\title{
Monomorphic ventricular tachycardia caused by arsenic trioxide therapy for acute promyelocytic leukaemia
}

\author{
${ }^{1}$ RA Ducas, ${ }^{2} \mathrm{MD}$ Seftel, ${ }^{3} \mathrm{~J}$ Ducas, ${ }^{4} \mathrm{C}$ Seifer \\ ${ }^{1}$ Cardiology Fellow; ${ }^{2}$ Oncologist; ${ }^{3}$ Cardiologist; ${ }^{4}$ Cardiologist, Department of Internal Medicine, University of Manitoba, Winnipeg, Manitoba, Canada
}

\begin{abstract}
Arsenic trioxide has become the treatment of choice for patients with acute promyelocytic leukaemia. Cardiovascular toxicity is known to occur with this therapy, in particular heart rhythm disorders due to QT interval prolongation. We present a case of ventricular arrhythmia with no QT prolongation in a patient receiving arsenic trioxide therapy.
\end{abstract}

KEYWORDS Arrhythmia, arsenic trioxide, promyelocytic leukaemia

DECLARATION OF INTERESTS No conflict of interests declared.

\author{
Correspondence to C Seifer, \\ Section of Cardiology, \\ Cardiac Sciences Department, \\ St Boniface General Hospital, \\ 40I Tache Avenue, Winnipeg, \\ Manitoba R2H 2A6, Canada \\ tel. + | 204258 I267 \\ e-mail cmseifer@sbgh.mb.ca
}

\section{CASE REPORT}

A 35-year-old woman presented to the emergency department with a 28-day history of intermittent, regular palpitations associated with pre-syncope. Throughout the previous 14 days the palpitations were increasing in frequency and duration. Two months prior to presentation, the patient was diagnosed with a relapse of acute promyelocytic leukaemia (APL) and salvage arsenic trioxide (ATO) therapy (7.9 $\mathrm{mg}$ intravenous daily) was initiated. On presentation, she was on day 34 of a planned 90-day course of ATO.

The patient had no significant prior cardiac history and there was no family history of cardiac disease. Blood pressure was $126 / 60 \mathrm{mmHg}$, and heart rate was 80 beats per minute. A 12-lead electrocardiogram (ECG) showed normal sinus rhythm with episodes of non-sustained monomorphic ventricular tachycardia (VT) with a corrected QT (QTc) interval of 443 ms (Figure I). Other relevant investigations included serum potassium $(4.3 \mathrm{mmol} / \mathrm{l})$ and magnesium $(0.92 \mathrm{mmol} / \mathrm{l})$ tests.

The patient was admitted for further management. Echocardiography was normal. Cardiac magnetic resonance imaging showed no delayed enhancement and normal right and left ventricular size and ejection fraction. Arsenic trioxide was discontinued and oral potassium, magnesium and metoprolol were initiated. One month after discharge, a 24-hour Holter monitor demonstrated no episodes of ventricular tachycardia. Metoprolol was gradually discontinued over the following three months and the patient has remained symptom free for the past 12 months.

\section{DISCUSSION}

Arsenic is a metalloid element with the atomic number 33 and has gained notoriety for its role in forming a number of poisonous compounds. In the form of

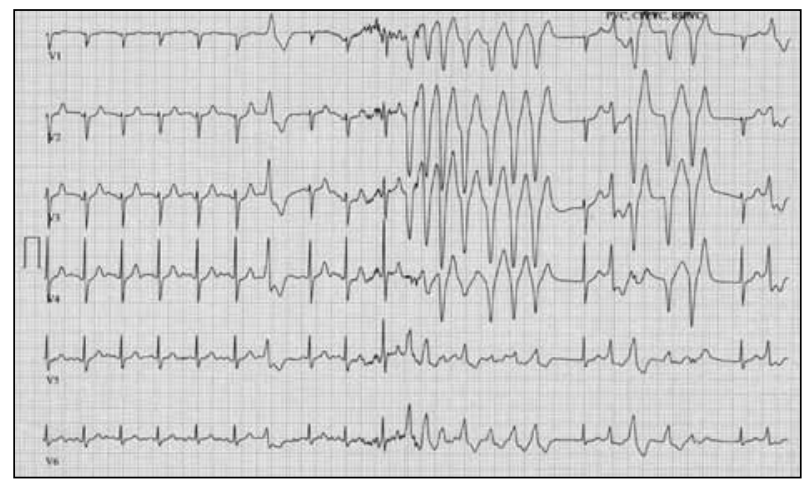

FIGURE I The patient's I2-lead electrocardiogram showed normal sinus rhythm with episodes of non-sustained monomorphic ventricular tachycardia with a corrected QT interval of $443 \mathrm{~ms}$.

inorganic compounds, arsenic has long been used medicinally. Traditional Chinese medicine has used arsenic compounds for millennia, based on the philosophy of using poison against poisonous disease. At the end of the nineteenth century and early twentieth century arsenic became a standard medication for the treatment of chronic myeloid leukaemia, primarily due to the lack of other efficacious options. However, with the advent of newer chemotherapeutic agents, arsenic fell out of favour around the 1950s.

The medical revival of arsenic is based on the treatment of APL.' Arsenic trioxide $\left(\mathrm{As}_{2} \mathrm{O}_{3}\right)$ induces high rates of complete remission in patients with relapsed APL. The mechanism by which $\mathrm{As}_{2} \mathrm{O}_{3}$ has its clinical efficacy is thought to be through the induction of partial differentiation and apoptosis in the leukaemogenic fusion protein PML-RARA, which makes it of particular use in APL. Arsenic trioxide has now become a standard drug in the treatment of both newly diagnosed and relapsed APL.' Tissue accumulation of arsenic occurs in the liver, kidneys, heart, lungs, hair and nails. On completion of ATO therapy, urinary arsenic excretion 
occurs over a prolonged time, with plasma arsenic levels declining to baseline approximately four weeks after the cessation of therapy.' This lengthy excretion time has implications for patient monitoring as tissue accumulation may result in delayed adverse effects.

Reported cardiovascular toxicities of ATO have begun to emerge as its use has increased in the treatment of APL. The majority of reported adverse cardiovascular effects are electrophysiological, including heart block, QTc prolongation, torsade de pointes and asystole. ${ }^{1-3}$ QTc prolongation is the most common, with an incidence of 26-93\%. ${ }^{2}$ Symptomatic torsade de pointes has been reported in $1-15 \%$ of case series treated with standard-dose ATO., ${ }^{1,3}$

The prolongation of the QTc interval appears to be due to a concentration-dependent blockade of $\mathrm{IKr}$ and $\mathrm{IKs}$ potassium currents compromising repolarisation and increasing electrical susceptibility to ventricular arrhythmias. ${ }^{4}$ Arsenic has also been shown to activate a time-independent IK-ATP which maintains normal repolarisation. The variability in QTc prolongation as well as the onset of ventricular arrhythmias likely represent competing activation and blockade of potassium channels during ventricular repolarisation. ${ }^{4}$ The susceptibility for ventricular pro-arrhythmia has been postulated to be linked to electrolyte abnormalities, specifically hypokalaemia and hypomagnesaemia, as well as concomitant treatment with QTc-prolonging medications.

In response to findings of arsenic cardiotoxicity, recommendations for cardiac monitoring have been set out by both the National Comprehensive Cancer Network in the US and the manufacturer of Trisenox ${ }^{\mathrm{Tm}}$. Prior to ATO therapy, a 12-lead ECG as well as serum electrolyte (potassium, calcium and magnesium) and creatinine tests are required. Pre-existing electrolyte abnormalities are to be corrected and an avoidance of QTc-prolonging drugs is encouraged. ${ }^{5}$ Recommendations for monitoring during active therapy include weekly ECGs for QTc measurements during induction therapy and before each treatment in post-remission therapy. For a QTc $>500 \mathrm{~ms}$, therapy should be withheld and the QTc reassessed with serial ECGs, along with electrolyte replacement, if indicated. Arsenic trioxide should be reinitiated when the QTc is $<460 \mathrm{~ms}$. The serum potassium concentration should be kept above $4 \mathrm{mmol} / \mathrm{l}$ and magnesium above $0.74 \mathrm{mmol} / \mathrm{l}^{.5}$

Our reported case of arsenic-induced VT demonstrates monomorphic VT which was not associated with a prolongation of the QTc or electrolyte abnormalities. The underlying mechanism of the arrhythmia may be due to an imbalance in the IK-ATP, IKr and IKs potassium channels during repolarisation, resulting in a normal QTc with increased susceptibility toVT due to a perturbation of normal repolarisation.

While the guidelines for monitoring patients on ATO therapy are centred on maintaining a QTc in the normal range as well as preventing electrolyte abnormalities that may be pro-arrhythmic, standard recommendations for monitoring may not be adequate in the absence of these risk factors.

\section{CONCLUSION}

To our knowledge this is the first documented case of symptomatic non-sustained VT caused by ATO with no QTc prolongation. This observation potentially supports more frequent cardiac monitoring in patients treated with ATO or the immediate investigation and possible withdrawal of ATO therapy in patients with symptoms of palpitations and pre-syncope.

\section{REFERENCES}

I Au WY, Kwong YL. Arsenic trioxide: safety issues and their management. Acta Pharmacol Sin 2008; 29:296-304. doi: I0. I I I I/j. I745-7254.2008.0077I.x

2 Yeh ET, Bickford CL. Cardiovascular complications of cancer therapy: incidence, pathogenesis, diagnosis, and management. J Am Coll Cardiol 2009; 53:223 I-47. doi:I0.1016/j.jacc.2009.02.050

3 Unnikrishnan D, Dutcher JP, Varshneya $\mathrm{N}$ et al. Torsades de pointes in 3 patients with leukemia treated with arsenic trioxide. Blood 2001; 97:1514-6. doi:10.1 182/blood.V97.5.1514

4 Drolet B, Simard C, Roden DM. Unusual effects of a QT-prolonging drug, arsenic trioxide, on cardiac potassium currents. Circulation 2004; 109:26-9. doi:I0.116I/0I.CIR.0000109484.00668.CE

5 O'Donnell MR, Appelbaum FR, Coutre SE et al. NCCN clinical practice guideline in oncology: acute myeloid leukemia. National Comprehensive Cancer Network; 20I0. Available from: http:// www.cancerplan.net/Best\%20Practise/aml.pdf 